\title{
Le mal-être féminin dans "Syngué sabour. Pierre de patience" d'Atiq Rahimi
}

\author{
Prof. adjoint: Hassan Elbahraoui \\ Professeur adjoint à la faculté des Lettres \\ Université de Benha
}

\section{Résumé}

Né en 1962 à Kaboul en Afghanistan, Atiq Rahimi est un écrivain, un réalisateur et cinéaste franco-afghan. Il vit à Paris depuis plus de 30 ans. Son quatrième roman "Syngué sabour. Pierre de patience" est publié en 2008. Il reçoit la même année "le prix Goncourt" et est traduit en de multiples langues. Désormais, Atiq Rahimi devient l'un des écrivains les plus lus en France et sa renommée commence à s'étendre partout. Il jouit sans conteste d'une réputation internationale.

Le roman contribue à la prise de conscience de la condition de la femme afghane et de sa situation socio-culturelle déplorable. C'est cette approche qui nous a permis de braquer la lumière sur le mal-être féminin qui met en lumière la marginalité sociale, le malheur et la douleur de la femme afghane. Prenant la défense de cet être malheureux, l'écrivain parle de son aliénation, de sa dépendance, de l'oppression conjugale, de sa dignité bafouée, de ses désirs frustrés et de ses secrets scandaleux.

La recherche présente analysera de près toutes formes du mal-être que la femme subie, parce que cette question est cruciale de nos jours en Afghanistan. Ce que nous avons voulu montrer dans ce travail, ce sont la dépendance incarnée par le mariage de force et la stérilité, l'asservissement de la femme au sein de la société afghane et ses tentatives de libération en racontant à son mari, étendu malade sur un matelas, ses secrets les plus intimes.

La société afghane est patriarcale. Elle légitime la domination que le statut masculin exerce sur la femme. Avant et après le mariage, la protagoniste du roman subit les conséquences fâcheuses et pénibles des droits dont dispose d'abord son père, puis son mari. De nombreux passages du récit dénotent une soumission totale de cette femme qui est maltraitée par un homme violent qui n'hésite pas à la battre pour la moindre futilité: cette personne est totalement soumise. On décide pour elle.

Le romancier nous présente avec beaucoup d'audace la réalité désastreuse vécue au quotidien par cet être victime des traditions. En plus, Le récit de Rahimi est un plaidoyer en faveur de la femme largement méprisée en Afghanistan. Les paroles, que la protagoniste croit prononcer dans un long monologue, dévoilent toute la vérité: L'héroïne du roman apprend à son mari plongé dans le coma, dans un long monologue, toutes les souffrances, les angoisses et les oppressions subies dans sa vie de femme. Ses secrets se succèdent sans cesse. Sans aucune retenue, sans aucun mensonge, elle nous révèle des secrets effroyables parce qu'inattendus venant de cette femme qui a vécu cloîtrée toute sa vie. 
Le chemin de l'exil d'Atiq Rahimi en France lui permet de ne pas sombrer dans le mutisme, de ressusciter la parole et de la rendre à la femme afghane. Et grâce à cette parole, la femme afghane se libère et arrive à atteindre une certaine délivrance. Son message fait résonner un cri de liberté pour l'avenir. Il y a peu d'espoir de liberté et d'avenir de la femme en Afghanistan où règnent la terreur, le fanatisme religieux, l'obscurantisme, l'oppression masculine et l'intolérance.

En effet, le récit rahimien est un prétexte pour prôner la liberté et déclarer la désobéissance aux interdits. Il fait présager d'une chance pour le dialogue égalitaire entre l'homme et la femme: il s'agit d'une solidarité utopique avec l'être méconnu, même plus méprisé, qu'est la femme.

Bref, Atiq Rahimi a écrit un roman à tendance réaliste qui a montré le mal-être féminin à un moment où l'Afghanistan n'est pas arrivé à franchir le seuil de la modernité. Pour ce, "évidemment on ne peut que saluer une œuvre aussi audacieuse que féministe".

\section{Le mal-être féminin dans "Syngué sabour. Pierre de patience" d'Atiq Rahimi}

Né en 1962 à Kaboul en Afghanistan, Atiq Rahimi est un écrivain, un réalisateur et cinéaste franco-afghan. En 1984, il quitte son pays natal miné par la guerre. Demeurer en Afghanistan, c'est être condamné à un avenir sans lendemain, c'est risquer de tomber entre les "mains des fondamentalistes qui détournent le désespoir de cette jeunesse à des fins religieuses extrémistes."

Il se réfugie donc au Pakistan pour un certain temps, puis demande protection auprès de l'ambassade de France à Islamabad. Quarante jours après, il jouit du statut de réfugié politique en France. Celle-ci l'aide à poursuivre ses études à Rouen. Après avoir terminé ses études en lettres modernes, il s'oriente vers les études audiovisuelles et obtient son doctorat de la Sorbonne. Sa thèse a pour titre "La fin dans le cinéma"2. Puis il s'engage dans la réalisation des films documentaires, tels "Zaher Shah", "Le royaume de l'exil" (2000), "Nous avons partagé le pain et le sel" (2001), "L'Afghanistan, un Etat impossible?" (2002), ainsi que dans l'écriture romanesque.

Vivant en France depuis une trentaine d'années, l'écrivain franco-afghan n'oublie à aucun moment son pays natal. Il écrit ses trois premiers romans en langue persane. Dans le premier roman, intitulé "Terre et cendres" ${ }^{3}$, il traite la terreur dont souffrent ses compatriotes pendant la guerre de 1979 à 1984. Ce roman est adapté par l'auteur lui-même au cinéma et son film reçoit le prix "Regard vers l'avenir" au festival de Cannes en 2004.

Le deuxième roman "Les Mille maisons du rêve et de la terreur" ${ }^{4}$ retrace les neuf jours et neuf nuits de marche que l'auteur parcourt avec 2000 afghanis avant d'atteindre le Pakistan. Dans son troisième roman "Le Retour imaginaire" ${ }^{5}$, il parle de son retour en Afghanistan après 28 ans d'exil en France et la chute des Talibans. 
Son quatrième roman "Syngué sabour. Pierre de patience" 6 , publié en 2008, reçoit la même année le prix Goncourt. C'est la première fois que le scrutin de ce prestigieux prix (sept voix contre trois) récompense un écrivain de cette zone du monde.

Désormais, Atiq Rahimi devient l'un des écrivains les plus lus en France et sa renommée commence à s'étendre partout. Le roman récompensé par le prix Goncourt est traduit en plusieurs langues. L'auteur jouit sans conteste d'une réputation internationale et Michel Nareau n'hésite pas à déclarer:

"L'auteur franco-afghan Atiq Rahimi a fait une entrée fracassante dans les milieux littéraires mondiaux." ${ }^{7}$

Adapté au cinéma, 4 ans plus tard, par l'auteur et Jean-Claude Carrière, il reçoit le prix du meilleur film au festival international des jeunes réalisateurs de Saint-Jeande-Luz en $2012^{8}$. Ce roman rahimien est "écrit à la mémoire de N.A. (Nadia Anjuman) - poétesse afghane sauvagement assassinée par son mari" ${ }^{19}$ en 2005. Placé sous le signe de la cruauté, il met en scène la destinée tragique de la femme afghane.

D'ailleurs, il est à souligner que le titre du roman est plutôt répété ou exprimé en deux langues : "Syngué sabour" en afghan veut dire " pierre de patience" en français. Le romancier entend par ce choix la pierre magique qui se trouve à la Mecque dans la maison de Dieu. Dans la légende perse, elle accueille les malheurs et les souffrances de ceux qui se confient à elle ${ }^{10}$.

Après ce tour d'horizon rapide et nécessaire, notre travail aborde uniquement le texte romanesque. L'action du roman se déroule à Kaboul en pleine guerre. La scène inaugurale nous montre un guerrier afghan étendu sur un matelas par terre, atteint d'une balle dans la nuque par l'un des combattants de sa milice. Il est plongé depuis trois semaines dans un coma profond. Sa femme s'installe auprès de lui, veille sur son corps immobile et le soigne avec des moyens rudimentaires ${ }^{11}$.

Les premières pages de "Syngué sabour. Pierre de patience" décrivent le cadre où se passe la majorité des évènements: il s'agit d'une chambre "vide de tout ornement."12 Abandonnée de tous, cette femme vit seule dans une chambre isolée, située à la frontière d'une ville ravagée par la guerre avec son mari agonisant. La solitude et la présence de la mort la remplissent d'une profonde angoisse qui la ronge et la rend quasi paranoïaque.

Dans cet isolement total, elle revoit les images de sa vie passée. Ces souvenirs ressuscitent en elle ses secrets les plus intimes et ses désirs les plus honteux. Le lecteur est témoin de ces évocations grâce à un monologue qu'elle extériorise croyant que son mari n'est qu'un corps inerte. A partir de ce moment, nous avons affaire à une des confessions les plus sincères. Il s'agit d'un étalage minutieux de l'état d'âme de cette femme afghane dont la vie n'est qu'une suite de souffrances. Prenant la défense de cet être malheureux, l'écrivain parle de son aliénation, de sa dépendance, de l'oppression conjugale, de sa dignité bafouée, de ses désirs frustrés et de ses secrets scandaleux.

Le roman contribue à la prise de conscience de la condition de la femme et de sa situation socio-culturelle déplorable. C'est cette approche qui nous a permis de braquer la lumière sur le malaise féminin qui met en relief la marginalité sociale, le malheur et la douleur de la femme afghane. 
Quoique " Syngué sabour. Pierre de patience" ait été étudié par la critique littéraire médiatique et universitaire, nous n'avons trouvé que quelques brèves analyses sur le malaise féminin. Il nous semble cependant que le sujet soit loin d'être épuisé. Un seul article, essentiellement issu d'approche féministe, comporte certaines pistes, tant théoriques que thématiques, que nous avons approfondies dans la présente recherche: "Ecriture au féminin par procuration. Pierre de patience d'Atiq Rahimi"13 de José Domingues de Almeida.

Notre recherche analysera de près toutes formes de malaise que la femme subie, parce que cette question est cruciale de nos jours en Afghanistan. Ce que nous avons voulu montrer dans ce travail, ce sont la dépendance incarnée par le mariage de force et la stérilité, l'asservissement de la femme au sein de la société afghane et ses tentatives de libération.

Le mot mal-être, tel qu'il est utilisé dans le cadre de cette recherche, n'est pas employé au sens psychiatrique du terme; mais il est dû à l'assimilation des différents aspects de la dépendance de la femme afghane.

\section{1-La soumission de la femme ou la domination masculine:}

A travers les aspects de la culture afghane, Atiq Rahimi dénonce un monde "irrespirable et hypocrite masculin dont les femmes (...) sont les premières victimes silencieuses." ${ }^{14}$ La société afghane est patriarcale. Elle légitime la domination que le statut masculin exerce sur la femme. Le rôle de celle-ci se limite à la procréation. Faire venir au monde des enfants, des mâles de préférence puisque les femelles sont mal accueillies à leur naissance. Ce système a fait de la femme un outil soumis à la volonté de son mari et à son bon plaisir.

\section{- Mariage de force}

La femme afghane est musulmane. Grâce à l'Islam, elle a acquis certaine droits: celui de vivre ${ }^{15}$, celui d'hériter et celui de la pratique religieuse. Mais l'Islam n'a quand même pas libéré totalement la femme. Celle-ci demeure inférieure à l'homme.

Avant et après le mariage, la protagoniste du roman subit les conséquences fâcheuses et pénibles des droits dont dispose d'abord son père, puis son mari. De nombreux passages du récit dénotent une soumission totale de cette femme qui est maltraitée par un père violent qui n'hésite pas à la battre pour la moindre futilité:

"Il (son père) cherchait n'importe quel prétexte pour nous battre (toutes les sept filles qu'il a)... il battait aussi ma mère." ${ }^{16}$

Ce qui intéresse ce père violent, ce sont ses cailles de combats. ${ }^{17}$ Une fois, il a dépensé tout son argent en achetant "une caille hors de prix." ${ }^{18}$ Il a passé des semaines et des semaines à la préparer pour un combat très important. Mais, "ironie du sort, il a perdu." ${ }^{19}$ Or, l'une de ses filles, à l'âge de 12 ans, devient "l'enjeu d'un pari." ${ }^{20}$ Son père, qui n'a "plus d'argent pour honorer le pari"'21, la donne à un homme de 40 ans.

L'héroïne du roman est effrayée de voir sa sœur aînée ainsi vendue. Elle se venge en se débarrassant d'une des cailles de son père. Mais la punition ne tarde pas et elle 
a été sauvagement battue. De plus, quand la protagoniste a atteint l'âge de 17 ans, une femme vient demander la main de sa sœur cadette pour son fils. Mais comme c'était le tour de l'héroïne, on la lui a proposée. Et la femme a accepté la marchandise:

"Bon, ce n'est pas grave, ça sera elle alors! En pointant son index charnu vers (elle) (...)."22

Le père de famille accepte donc "sans hésitation une seule seconde."23 La jeune fille est totalement soumise. On décide pour elle et elle doit accepter les fiançailles qui ont lieu sans le fiancé présent au front. Ce futur mari est seulement un nom pour tout le monde. Il n'est qu'un nom, un personnage absent pour cette jeune fille.

D'un commun accord, le mariage aura lieu dans un an. La guerre n'ayant pas pris fin, le mariage ne sera pas remis. La cérémonie de mariage est effectuée sans le mari. Ce sont la photo et le foutu kandjar de ce dernier qui prennent sa place à côté de sa future épouse.

On la marie ainsi avec un homme absent sans qu'on lui demande son avis. Elle se marie avec un guerrier inconnu qu'elle n'a jamais vu et qu'elle verrait trois ans après le mariage:

"Fiancée pendant un an et mariée pendant trois ans à un homme absent." 24

Désormais, cette jeune épouse doit attendre l'arrivée de son mari. Elle n'a pas le droit de voir ni sa famille, ni ses copines, car une jeune fille mariée, encore vierge, ne doit pas "fréquenter les autres filles mariées." ${ }^{25}$ Elle ne dort pas dans sa chambre, mais avec sa belle-mère qui veille sur sa chasteté.

A l'intérieur du foyer, elle s'occupe des travaux les plus harassants. Ses beauxparents disposent totalement d'elle. Trois ans d'attente, souffrant d'une solitude effroyable, la jeune mariée a essayé durant cette longue période de tracer une image de ce conjoint absent, mais une angoisse terrible remplit son âme.

Un jour inattendu, ce guerrier revient. Il s'assoit à côté d'elle sans rien dire. Tout d'abord, elle le voit, le regarde "à la dérobée" ${ }^{26}$, puis elle le contemple "dans le moindre mouvement de (son) corps, dans la moindre expression de (son) visage." ${ }^{27}$ Ensuite, elle jette un long regard sur lui. Mais cet homme a "l'air absent, arrogant." 28 Il ne la voit ni lui parle plus: "pas un mot, pas un regard..."29 ; de plus, il est ailleurs et ses yeux sont rivés sur nulle part. C'est ce que suggère la parole des sages à cet égard:

" Il ne faut jamais compter sur celui qui connait le plaisir des armes!"30

Cette femme afghane tremble de peur à cet instant. Elle éprouve une frayeur terrible du lit et du sang. Mais ce genre de peur ne l'éloigne pas de son plaisir. Au contraire, elle jouit des moments d'amour qu'elle partage avec son mari. Et petit à petit, son unique tâche est de se vouer à son mari, de se consacrer totalement à ses beaux-parents et même de veiller, plus tard, sur ses enfants. La jeune épouse est coupée du monde extérieur. Elle ne sort pas de la maison et ne rend pas visite à sa famille. Elle est privée de ses droits et de sa liberté en tant qu'être humain.

Si elle sort, elle se fait accompagner de sa belle-mère par honneur et par sécurité. Or, elle se voile: 
"Au seuil de la porte, $(.$.$) elle se penche pour attraper son voile." { }^{31}$

Même "son corps est enveloppé dans une robe longue." ${ }^{32}$ C'est parce que "le voile fait partie des traditions vestimentaires nationales (... $)^{\prime 33}$, qu'il existe depuis longtemps, qu'il n'a pas disparu et qui s'est plutôt renforcé, il fait partie de la tradition religieuse qu'il faut sauvegarder.

Cette femme n'a jamais pu parler à son époux qui ne l'a jamais embrassée. Celui-là la quitte souvent. Il n'a pas supporté l'appel des armes. Ces dernières incarnent tout pour lui: la mère, la sœur, l'honneur ou comme l'affirme l'héroïne ainsi:

"Vous les hommes! Quand vous avez des armes, vous oubliez vos femmes." 34

La jeune mariée est rongée par l'inquiétude et attend avec angoisse son retour:

"J'avais l'impression que quelque chose manquait. Non pas dans la maison, mais en moi... Je me sentais vide." 35

\section{- La stérilité de la femme}

D'autre part, le roman rahimien est parfois porteur d'une réflexion sur la stérilité du personnage féminin. La femme stérile souffre doublement. Elle se voit maltraitée et vit en marge de la société. La tante de la protagoniste en est l'exemple le plus éloquent. Isolée, l'héroïne n'est entourée d'aucune figure féminine. Toutefois, le romancier fait allusion quelque fois à une tante chez qui elle laisse ses deux petites filles à l'époque de la guerre. ${ }^{36}$

Presque invisible le long du texte, cette vieille femme semble envahir la mémoire de la protagoniste. Seule, délaissée et presque répudiée à cause de sa stérilité, la tante extériorise parfois sa haine envers l'Homme tyran. Cette femme que la nature a défavorisée, mène une vie plus malheureuse que ses consœurs. Elle s'occupe uniquement des travaux de la maison, ne sort pas, et est plus séquestrée que les autres. C'est une femme qui se sent terriblement seule:

"Deux ans de mariage ont passé et ma tante n'a pas pu enfanter pour lui. (...). Bref, ma tante était stérile. Autrement dit: bonne à rien." ${ }^{37}$

Sa stérilité lui cause beaucoup de problèmes, surtout vis-à-vis de son époux. Les relations conjugales sont des relations de haine et de méfiance. Aucune intimité, mais plutôt des outrages, des humiliations et surtout de la violence.

La tante est une femme malheureuse, soumise et totalement écrasée par son mari. Elle n'a aucun droit, même pas celui de parler, et cela dure depuis longtemps. Son mari la méprise et la bat durement, car il perd l'espoir d'avoir un jour des enfants d'elle et est probablement attiré par une autre. Or, il "l'a envoyée en province chez ses parents pour les servir." ${ }^{38}$

Très belle, son beau-père l'a fréquemment violée, ce qui démontre la corruption cachée sous les apparences respectueuses. Un jour, elle a refusé et "lui a fendu le crâne." ${ }^{39}$ Par conséquent, elle a été chassée de la maison par les parents de son mari. 
Puis, elle a été rejetée par son époux. Or, elle souffre terriblement de la répudiation forcée. Elle retourne chez sa propre famille, précisément chez son frère, le père de la protagoniste. Mais, malheureusement, celui-ci l'a aussi abandonnée.

Devant cet abandon familial, elle n'a qu'un seul chemin à suivre: feindre la mort pour fuir la honte de sa stérilité et les abus de sa famille, et surtout aller vivre ailleurs où personne ne pourrait la reconnaitre: "elle va choisir de passer pour morte et fuir." 40

Un passage du roman est exemplaire à cet égard:

"Alors elle, la "sale tache" de la famille a disparu, laissant un mot pour dire qu'elle avait mis fin à sa vie."41

La tante est victime de l'ordre social existant. Elle est exclue de la famille en raison de sa stérilité, et finit par vivre dans "une maison close." ${ }^{42}$ Seule, elle peut difficilement gagner sa vie autrement. Elle se prostitue, bien qu'elle ne soit pas une femme de milieu défavorisé. Elle a recours à ce moyen de subsistance. C'est à travers la parole de la protagoniste qu'on apprend que sa tante devient prostituée. ${ }^{43}$ Mais le roman ne comporte aucune scène descriptive de son rapport avec la prostitution.

Le cas de la tante n'est pas rare dans la société afghane. Celle-ci incarne l'image d'une certaine catégorie de femmes rejetées par la société, privées de leurs droits, à la merci de ceux qui les accueillent et les asservissent.

L'héroïne du roman risque pour un certain temps de partager le sort de sa tante. Cinq ou six mois ont passé sans aucun indice de grossesse. ${ }^{44}$ Là, le romancier dévoile avec beaucoup de finesse et d'acuité l'état d'âme de cette jeune femme affolée à l'idée de subir le même sort que sa tante.

Pour matérialiser la condition de vie désastreuse de la femme afghane, le romancier excelle dans le choix des situations inhumaines employées à cet être dont la faute essentielle et unique est d'être née une "femelle".

\section{$\underline{\text { 2- L'asservissement de la femme }}$}

Le mari de la protagoniste est un moudjahidin qui "s'était battu sur tous les fronts." 45 Il reçoit une balle dans "une bagarre minable avec un type - de son propre camp, d'ailleurs - qui avait dit: je crache dans la chatte de ta mère! Juste pour une insulte!"46 Par conséquent, ce guerrier devient grabataire dans une chambre de sa maison. Couché sur un matelas et caché à tous, gravement blessé dans la nuque, il est plongé dans le coma. Le romancier s'attarde à décrire ce mari agonissant. Les termes utilisés tels "les jambes cadavériques"47, "la peau pâle. Pleine de rides", "ses bras inertes"48, "cet air hagard", "regard perdu"49 sont très expressifs et sont annonciateurs d'une fin prochaine. Il ne bouge pas. Il demeure inerte et insensible: aucun signe de vie, "aucune douleur, aucune souffrance" ${ }^{50}$ et aucune plainte. Il n'est qu'un cadavre vivant, car il respire encore.

Auprès de lui, sa jeune épouse veille sur lui et "reste à son chevet." ${ }^{51}$ On devine le désarroi qui l'agite. Campagne fidèle et docile, elle ne manque jamais à ses devoirs. Elle le soigne et le sert, se met complètement à sa disposition, ne néglige aucun détail. Elle a des travaux quotidiens à accomplir: chaque matin, la poche de perfusion s'épuise 
et la femme apporte deux verres d'eau: "l'un pour la poche de perfusion, l'autre pour humecter les yeux de l'homme." ${ }^{52}$ Elle instille même des gouttes de collyre aux yeux de son mari.

La même opération se répète inlassablement le soir. De plus, elle part de la chambre pour revenir avec une bassine en plastique, une serviette, un drap propre et des vêtements. Elle lave son homme, le change et le réinstalle à sa place. ${ }^{53}$ Mais dans cette description détaillée, il n'est pas question de sentiments; la femme fait tout pour son mari en suivant sans conteste les coutumes religieuses de la société afghane.

Rien d'autre n'arrive que ces travaux quotidiens effectués par cette jeune mariée. De surcroit, l'appel à la prière à intervalles réguliers la pousse à sortir de sa torpeur. "Elle prend le petit tapis, le déplie et l'étale par terre." ${ }^{54}$ La prière faite, elle est assise sur place en lisant quelques versets du Coran. Elle pleure à chaudes larmes et prie Dieu de protéger son mari et de le guérir en répétant avec un cri étouffé:

" Dieu, fais qu'il revienne à la vie." 55

Elle récite inlassablement "quatre-vingt-dix-neuf fois par jour l'un des quatre-vingtdix-neuf noms de Dieu." ${ }^{56}$ Citons par exemple, au sixième jour, elle se met à égrener son chapelet en répétant sans cesse "al-Qahhâr, Le Dominateur." ${ }^{17}$ Cette héroïne ne relâche pas son chapelet noir tenu par la main et ce nom de Dieu résonne partout dans la maison: dans la pièce près de son mari, contre la fenêtre, dans le couloir et derrière la porte. Ainsi, la journée égale-t-elle pour elle "quatre-vingt-dix-neuf tours de chapelet." ${ }^{58}$ Et elle effectue la même stratégie chaque jour avec un autre nom de Dieu.

"Atiq Rahimi nous présente (ainsi) le portrait d'une femme dévouée à son mari blessé (...)." ${ }^{59}$ Celle-ci semble délaisser complétement ses enfants qui ne sont pas en âge de pouvoir se débrouiller toutes seules.

L'action du roman se déroule tout au long du récit presque dans la chambre qui abrite l'homme blessé et sa jeune épouse. A l'intérieur, la mort rôde. Dehors, le quartier est livré à la guerre civile qui fait rage et dont "la femme suit les évènements (...) de la fenêtre. ${ }^{60}$ Celle-ci constitue le trait d'union entre l'intérieur et l'extérieur. Mais il est à remarquer que les deux espaces (fermé et ouvert) sont tous les deux placés sous le signe du danger. La mort est partout et semble n'épargner personne.

Les rues en chaos sont soumises aux coups perpétuels de feu, surtout le soir, qui empêchent les gens de sortir:

"On tire de tous les côtés, dans tous les sens." ${ }^{61}$

Ou encore: "Dans la ville, on tire toujours. De loin, de près, sporadiquement." 62

On entend partout des déflagrations violentes des bombardements qui font "trembler la terre" ${ }^{63}$ et dont les souffles détruisent les vitres. Or, les rues sont réduites en poussières. Elles semblent être enfumées, car "la fumée et l'odeur de la poudre prolongent leurs souffles." ${ }^{64}$ La suie couvre tout. Elle envahit toutes les maisons, toutes les cours et s'épand dans la pièce de l'homme couvert de poussière noire alors que sa femme lui essuie le visage pour enlever cette suie. ${ }^{65}$ 
Dans cette atmosphère infernale, le romancier dénonce les dessous d'une société minée par l'égoïsme et les tares incurables. Au moment du danger, chacun cherche sa propre sécurité et son propre intérêt. Aucun partage, aucun sentiment sincère n'anime les membres de cette grande famille: parents, frères, sœurs prennent la fuite devant ce danger imminent, laissant leur frère agonissant et sa petite famille affronter un sort terrible:

" Où sont tes frères qui étaient si fiers de te voir te battre contre leurs ennemis." 66

Ou encore: "Tes frères aussi (...) sont tous partis. Ils nous ont abandonnés! Les lâches! Ils ne m'ont pas emmenée avec eux parce que tu étais vivant! (...)." 67

Devant cette situation, la protagoniste les condamne, car ils la laissent toute seule sans protection avec son mari impuissant et ses enfants. Quoiqu'enfermée dans une chambre de malade, l'héroïne a accès au monde extérieur grâce à la fenêtre. Elle voit et nous fait voir ce qui se passe dans la rue, lieu de combat et de violence. Un tableau sinistre s'étale devant nos yeux: les cadavres jonchent la rue et sont la proie des chiens errants qui les dévorent sauvagement; les maisons s'écroulent sous les bombardements, le feu est partout. Ces scènes violentes sont l'expression d'une condamnation que le romancier adresse aux responsables et aux chefs d'Etat.

Tout au long de cette guerre civile, la mort est omniprésente. L'héroïne est choquée et attristée quand elle découvre des voisins assassinés. La sécurité disparait totalement. Or, elle veut à tout prix défendre et soutenir ses deux petites filles. Elle les emmène loin de son quartier de combat et les laisse chez sa tante à l'autre bout de la ville:

" Et aujourd'hui, c'est cette tante qui m'a recueillie. Elle aime mes enfants. Et les filles l'aiment aussi." 68

Dès lors, elle revient dès les premiers rais du jour passer toute la journée avec son mari, puis elle l'abandonne temporairement le soir pour se réfugier dans "un endroit plus sûr"69 chez sa tante.

Les hommes s'entretuent comme des bêtes sauvages. Deux camps du djihad, déjà luttant ensemble contre les Russes, deviennent des frères ennemis. Ils se livrent des combats féroces et les civils innocents paient la facture de leur violence. Rien n'est sacré pour eux. Tout leur est permis. Ils assaillent les maisons et s'en servent comme abri sans tenir compte des habitants. Les combattants ont occupé la maison de la protagoniste toute une nuit en attendant l'arrivée de l'aube. ${ }^{70}$

Il apparait que le peuple afghan "se bat contre lui-même." ${ }^{71}$ Il en résulte l'extermination des êtres humains:

"Au-dehors, quelque part, pas très loin, quelqu'un tire une balle. Un autre, plus proche, riposte. Le premier tire une deuxième balle. L'autre ne répond plus." 72

De plus, la ville est détruite, ravagée en ruines par ces combats interminables entre deux camps luttant. Les gens, même le mollah, ne sortent pas de chez eux, ayant "peur 
des balles perdues." ${ }^{73}$ Le dehors de la maison n'est qu'une menace aveugle. Il y a aussi des patrouilles dans chaque coin de la ville. Les moudjahidines "fouillent toutes les maisons." 74

Ne pouvant riposter contre cette invasion, l'héroïne du roman fait de son mieux pour se cacher et cacher son mari aux yeux de ces êtres barbares. Elle se met, le plus vite possible, à préparer une cachette pour son mari derrière le rideau vert. Sa ruse réussit, car les combattants, qui entrent et explorent la chambre, s'évadent de la maison sans croiser ce "mari impuissant à se défendre." 75

Disons que le moment de cette guerre ravagée est assiégé de tant de terreur, de censure, d'obscurantisme, d'enfermement, d'aliénation pour la femme afghane. De même, il est à noter que le romancier fut un témoin oculaire de cette guerre fratricide qui n'a laissé que ruines et désolation, flammes et sang.

Après l'évacuation, la vie reprend son cours dans la chambre du malade. La femme de ce dernier est dans un état de prostration et d'épuisement. Elle est à bout de forces, demande à son mari de ne pas la laisser seule avec ses petites filles, car elle n'a que lui:

"Sans toi, je ne suis plus rien. Pense à tes filles! Qu'est-ce que je vais faire avec elles? Elles sont si petites..."76

Un silence total suit ses prières. Le mari ne réagit pas. Elle le secoue. Aucune réaction. "Elle appuie encore plus fort. Aucune plainte. Ni dans les yeux ni dans le souffle." ${ }^{77}$ Elle attend vainement qu'il fasse signe de vie. Cet homme "est toujours là dans la même position. ${ }^{178} \mathrm{Il}$ est toujours inerte, froid et impassible. Et l'auteur du roman décrit la peur, l'affolement et l'angoisse de l'héroïne.

Rien n'arrive "que des respirations." ${ }^{79}$, que le silence qui envahit toute la pièce. Elle est soumise à un destin malheureux avec son époux placide. "Une inquiétude mêlée de trouble, se lit dans son regard." ${ }^{10}$ Accablée de routine quotidienne, elle n'achève pas totalement ses travaux quotidiens très durs, car ses forces "défaillent de jour en jour." ${ }^{81}$

A force de ne vivre que pour son mari, auprès de lui, avec le rythme de sa respiration, la femme afghane lui arrive des fois de désirer la mort pour son mari:

"Si...tu avais été mort, les choses auraient été différentes." ${ }^{82}$

Cette femme s'indigne contre l'état de maladie incurable de son mari. Elle est en émeute et laisse éclater sa colère discrète:

"Sa rage cherche sa voix dans la gorge. (...). Un sanglot lui noue la gorge, et ne laisse sortir qu'un cri étouffé." ${ }^{83}$

Atiq Rahimi présente ici une femme ou plutôt la femme afghane "bafouée par des années de soumission et d'outrages" ${ }^{84}$, par "le fanatisme religieux, l'intolérance et l'oppression." 85 Le romancier nous présente avec beaucoup d'audace la réalité désastreuse vécue au quotidien par cet être victime des traditions.

Devant le mutisme du corps gisant de son mari, l'épouse se déchaine: 
"Il me rend folle! Il me rend faible! Il me pousse à la parole! à avouer mes fautes, mes erreurs! Il m'écoute! Il m'entend! C'est sûr!"86

Et importée par une violence indomptable, elle se laisse aller à une confession effrayante:

" Dans une société afghane où la femme n'est qu'un objet presque sans âme, l'épouse se retrouve dans une situation où elle peut enfin dire tout ce qu'elle a sur le cœur sans que son mari puisse réagir." ${ }^{87}$

À côté de son époux mourant, elle nous livre ses secrets les plus honteux, ses regrets et ses remords. Et très vite, les souvenirs l'emportent.

\section{$\underline{\text { 3-La confession de la femme }}$}

En 1984, Atiq Rahimi quitte l'Afghanistan " où la parole trop souvent souffre sous les régimes dictatoriaux, et où, depuis 40 ans, la censure est toute puissante." 88 Et le chemin de son exil en France lui permet de ne pas sombrer dans le mutisme, de ressusciter la parole et de la rendre à la femme afghane.

Le récit de Rahimi est un plaidoyer en faveur de la femme largement méprisée en Afghanistan. Les paroles, que la protagoniste croit prononcer dans un monologue, dévoilent toute la vérité. Sans aucune retenue, sans aucun mensonge, elle nous révèle des secrets effroyables parce qu'inattendus venant de cette femme qui a vécu cloîtrée toute sa vie. Et la femme afghane ressemble à toutes les autres femmes du monde avec ses faiblesses et ses grandeurs. Dans une grande partie du récit, le lecteur va de surprise en surprise, témoin de cette confession terrible. Sûre de l'inertie de son mari, la jeune épouse raconte ses secrets et ses désirs les plus intimes. Même plus, elle lui confesse ses péchés.

Dès lors, l'héroïne du roman prend en charge d'évoquer son passé avec tous ses détails. "Elle prend la parole pour combler le silence" ${ }^{89}$, pour bercer son chagrin et son amertume, alors que de sporadiques fusillades résonnent en dehors autour d'elle. Elle apprend à son mari, dans un long monologue, toutes les souffrances, les angoisses et les oppressions subies dans sa vie de femme.

Mais cet homme, réduit à un profond coma et au silence, n'est pas au fait inconscient. Il entend et ces révélations terribles ne sont qu'une punition méritée semble dire le romancier. Bafoué dans son honneur, il ne peut guère changer la réalité humiliante. Devant ces pages compactes, le lecteur ne peut s'empêcher de poser de multiples questions. Dans quelle mesure la parole de la protagoniste exprime-t-elle ses blessures, sa lassitude, ses souffrances, ses angoisses et son exaspération? Dans quelle mesure cette parole nous renvoie à un aspect libérateur au fil de la lecture? Cette confession libère-t-elle la femme de ses remords? Arrive-t-elle à prendre sa revanche? Comment le discours progresse-t-il dans cette œuvre romanesque?

Tout d'abord, cette jeune épouse hésite avant de se lancer dans cette voie périlleuse. Elle "respire profondément pour dire un mot. Elle n'y arrive pas. Le mot doit être lourd, lourd de sens, lourd à écraser sa voix. Elle le garde alors au fond d'elle." ${ }^{10}$ Mais, petit 
à petit, elle surmonte sa timidité. Et d'un ton désolé, elle s'encourage enfin pour dire à son époux quelques mots: " Je ne peux rien faire pour toi. Je crois que tout est fini!"191

De plus, cette mère se livre pour la première fois et étale devant ses yeux sa vie de sacrifices et d'humiliations. Pour la première fois, elle ose lui adresser des reproches:

" "Et toi, tu savais que tu avais une femme et deux filles!" Elle se frappe sur le ventre. Une fois. Deux fois. Comme pour expulser ce mot lourd qui s'est fui dans ses tripes. Elle s'accroupit et crie: "est-ce que tu pensais un moment à nous lorsque tu épaulais ta putain de Kalachnikov? Fils de ...."92

Premières reproches, premières révoltes, la jeune femme se lance dans une accusation que personne ne peut contredire, ni arrêter. Le premier pas fait, le pas le plus difficile accompli, tout s'enchaîne et l'on assiste à une avalanche de secrets qui se précipitent avec un rythme effrayant. Et le plus honteux, le plus infâme, monte à la surface pour éclabousser toute une vie jusqu'ici respectable, comme on le croyait.

Du premier coup, elle tremble en lui affirmant que ses frères ont toujours l'habitude de la surveiller par la fenêtre du hammam, en se branlant, au moment où elle se lave. En plus, "ils ont toujours envie de (la) baiser! (...) tout le temps, durant les trois ans de son absence..." ${ }^{93}$ La jeune épouse le dit sans oser regarder son homme ou bien en ayant un regard qui chercher quelque chose par terre. Et devant cette première confession, elle hésite:

" Qu'est-ce que je dis? Pourquoi je dis tout cela?! Mon Dieu, aidemoi! Je n'arrive plus à me contrôler. Je dis n'importe quoi..."94

A ce moment-là, la femme s'efforce de se taire. Mais elle n'y arrive pas. Ravagée par ses souvenirs et contre toute attente, elle se libère et raconte à son mari "tout ce qu'elle n'a jamais osé ou pu lui dire." ${ }^{95}$ Sa langue se délie et revit la nuit de ses noces avec ses moindres détails. Nuit passée sous le signe de la violence symbolisée par le sang. Cette mariée n'avertit pas son mari qu'elle a son écoulement mensuel dans cette nuit. Mais lui, il se sent content et fier en voyant le sang, signe de la virginité. ${ }^{96}$

Le passé revit devant nous dans ses moindres détails. C'est ainsi qu'elle évoque le rôle déterminant qu'ont joué sa tante et son beau-père dans sa vie. Celui-ci était malade à la fin de sa vie. C'est la protagoniste seule qui s'occupait de lui. Durant les longues heures qu'elle passait auprès de lui, il n'était question que d'une pierre magique, une "pierre noire, précieuse." ${ }^{97}$

Dans la légende perse, Syngué sabour est le nom d'une pierre noire de patience: il s'agit d'une pierre magique que l'homme pose devant lui et lui confie tous ses secrets, ses détresses et ses angoisses. Grâce à ces confessions, la personne se délivre du poids de ses péchés:

"Tu sais, cette pierre que tu poses devant toi...devant laquelle tu te lamentes sur tous tes malheurs, toutes tes misères... à qui tu confies 
tout ce que tu as sur le cœur et que tu n'oses pas révéler aux autres...(...). "Tu lui parles, tu lui parles. Et la pierre t'écoute, éponge tous tes mots, tes secrets, jusqu'à ce qu'un beau jour elle éclate. Elle tombe en miettes." (...). "Et ce jour-là, tu es délivré de toutes tes souffrances, de toutes tes peines..."98 Dit-il le beau-père à l'héroïne du roman.

Cette pierre noire est aussi celle qui se trouve à la Mecque et "autour de laquelle tournent des millions de pèlerins durant la grande fête de l'Aid."99 On voit bien que l'écrivain aussi bien que son héroïne sont obsédés par l'idée de délivrance obtenue par la confession sincère et totale.

Dans cette scène capitale, le mari est "le confident muet et forcé" 100 de cette femme afghane. Or, celle-ci fait de lui sa syngué sabour. Elle lui confie tous ses secrets dans l'espoir d'être absoute de ses fautes:

" Je vais tout te dire, ma syngué sabour, tout. Jusqu'à ce que je me délivre de mes souffrances, de mes malheurs, jusqu'à ce que toi, tu..."101

Ses secrets se succèdent sans cesse. Elle a tant de choses à lui raconter. Plus rien ne l'arrête et "à chaque fois que l'histoire se termine, une autre débute." ${ }^{102}$ Elle défie tous les tabous. C'est ainsi qu'elle lui parle impudemment d'une scène d'amour ${ }^{103}$ qui l'a réunie avec un jeune garçon âgé de seize ans. Celui-ci était un des guerriers qui sont entrés un jour fouiller dans cette pièce fermée. ${ }^{104}$ Le lendemain, cette jeune épouse se retrouve seule avec ce jeune homme qui la menace de son fusil. Obligée tout d'abord à se soumettre aux désirs de ce jeune soldat, elle prend goût à ces caresses et nous assistons à une scène pornographique des plus osées.

Les rencontres se multiplient et le plaisir se renouvelle et s'intensifie. ${ }^{105}$ D'ailleurs, elle accuse son mari de son incapacité de protéger son honneur:

" Tiens, voilà ton honneur baisé par un jeune de seize ans! Voilà ton honneur qui baise ton âme!"106

Mais, après cet aveu, elle réalise la monstruosité de ce qu'elle vient de dire:

" Ce n'est pas moi. Non, ce n'est pas moi qui parle...c'est quelqu'un d'autre qui parle à ma place...avec ma langue. Il est entré dans mon corps...Je suis possédée. J'ai vraiment une démone en moi. C'est elle qui parle. C'est elle qui fait l'amour avec ce garçon." ${ }^{107}$

D'ailleurs, les souvenirs s'accumulent, de temps en temps, à la mémoire de cette protagoniste. Le moment est arrivé de dévoiler son dernier secret pour son homme musulman. Ne pouvant plus rebrousser chemin, elle s'enhardit jusqu'à lui avouer qu'il n'est pas le père de ses deux petites filles: 
" Oui, ma syngué sabour, ces deux filles ne sont pas les tiennes! Et tu sais pourquoi? Parce que c'était toi qui étais stérile, pas moi!"108

Cinq mois après son mariage, la mère des deux petites filles s'attarde à se procurer un enfant de son mari. Sa belle-mère, comme les autres membres de la famille, lui reproche d'être stérile. ${ }^{109}$ Elle suggère, de temps à autre, une autre épouse à son fils. Et pour ne pas être comme sa tante, elle va, en compagnie de sa belle-mère, rencontrer un "sage Hakim"110 pour obtenir ses talismans. Son époux "connai(t) l'histoire, mais pas la vérité." ${ }^{111}$ Ce sage n'est que le maquereau de sa tante. Il l'enferme dans une chambre sombre avec un jeune homme très fort. Et la femme aurait dû coucher plusieurs fois avec celui-ci pendant quelques séances successives jusqu'au moment où elle tombe enceinte.

Cette ultime confession, comme celle qui la précède, est une transgression des tabous sexuels proscrits par les coutumes musulmanes, car "oser la parole, c'était provoquer le diable et la malédiction." ${ }^{112}$

Le miracle est arrivé. La syngué sabour ou la pierre de patience est menée au terme désiré: après avoir absorbé toutes les confidences de la femme, elle se brise et lui apporte la délivrance. Cette révélation finale secoue le guerrier musulman immobile. Il revient à la vie, a du mal à accepter la réalité et ne domine pas sa réaction violente:

"(Il) l'attrape par le poignet. (...). Il se dresse brusquement telle une roche, raide et sèche, que l'on soulève d'un seul coup. (...). (il) l'attire à lui, attrape ses cheveux et envoie sa tête cogner contre le mur. (...). Lui, visage terne et have, agrippe à nouveau la femme, la soulève et la projette contre le mur où le kandjar et la photo sont accouchés. Il s'approche d'elle, la saisit encore, la hisse contre le mur. La femme le regarde avec exaltation. Sa tête touche le Kandjar." ${ }^{113}$

Par geste de défense, la femme s'empare du kandjar et "l'enfonce dans le cœur de l'homme. Pas une goutte de sang ne jaillit."114 Ensuite, le corps de l'épouse traîne par terre, le mari frappe sa tête contre le sol et lui tord le cou d'un mouvement sec. ${ }^{115}$ Elle subit des sacrifices au prix de sa liberté d'expression. En tentant de défendre son autorité et les traditions religieuses, cet époux afghan reçoit un kandjar enfoncé dans le cœur. On ne sait plus si tous les deux personnages sont décédés ou non. Mais, probablement ils n'ont pas succombé à leurs blessures:

"La femme expire.

L'homme inspire.

La femme ferme les yeux

L'homme demeure les yeux égarés.

Quelqu'un frappe à la porte.

L'homme, le kandjar fiché dans le cœur, va s'allonger sur son matelas au pied du mur, face à sa photo.

La femme est écarlate. Ecarlate de son propre sang.

Quelqu'un entre dans la maison. 
La femme rouvre doucement les yeux."116

Ce récit n'a pas de conclusion précise. Le romancier laisse le lecteur imaginer la fin qu'il voit probable. C'est ce qu'il explique en disant:

" Je vais contre toute la dramaturgie, contre tous les codes, ce qu'on appelle "le code de la clôture". Il n'y a pas de fin véritable dans Syngué sabour. (...). Tout le monde doit trouver sa fin. C'est important pour moi que les lecteurs cherchent la fin." ${ }^{117}$

Il découle de cette ultime révélation une sorte de châtiment par la perte de la vie de la femme aussi bien que l'espoir d'un possible déliement d'un péché. Il parait que cette protagoniste fonde un monologue audacieux "qu'une femme ne pourra(it) jamais avouer dans un monde machiste et obscurantisme comme celui de l'Afghanistan (...)." ${ }^{118}$ Le roman rahimien fait présager d'une chance pour le dialogue égalitaire entre l'homme et la femme. "On y entrevoit la possibilité d'une réconciliation et d'une solidarité renouvelées des genres(...)."119

Grâce à la parole, la jeune femme se libère et arrive à atteindre une certaine délivrance. Son message fait résonner un cri de liberté pour l'avenir. Il y a peu d'espoir de liberté et d'avenir de la femme en Afghanistan où règnent la terreur, le fanatisme religieux, l'obscurantisme, l'oppression masculine et l'intolérance. Les oiseaux migrateurs, qui sont "figés dans leur élan" ${ }^{120}$, se réunissent et s'envolent vers la liberté:

"Quelqu'un entre dans la maison. La femme rouvre doucement les yeux. Le vent se lève et fait voler les oiseaux migrateurs au-dessus de son corps." 121

C'est-à-dire, "Syngué sabour. Pierre de patience" d'Atiq Rahimi est un prétexte pour prôner la liberté et déclarer la désobéissance aux interdits. Dans ce texte, le romancier s'inscrit dans cette tendance nouvelle, pleine d'espoir. Il tient à s'expliquer. S'il a écrit son texte en français, c'est parce qu'il ne pouvait faire autrement:

"Ma langue maternelle, le persan, m'impose des tabous, des interdits. (...). Adopter une autre langue, le français, c'est choisir la liberté. On ne se marie pas avec sa mère! Avec le français, j'étais libéré de tonnes de contraintes affectives."122

Ou encore: " Le français m'a permis de me libérer, de transgresser les tabous." 123

Comme le déclare l'écrivain lui-même, ce "roman n'a pas de réelle présence" $^{124}$ dans son pays natal. L'écriture romanesque est fondée, en occident, sur la notion d'individu. Mais, cet être humain, notamment la femme, n'a pas le droit d'exister dans la culture afghane. Il n'a pas de valeur et se définit 
par rapport à sa société ou encore à sa communauté. C'est pourquoi, on s'acharne à écrire des poèmes en Afghanistan. On s'y cache, on n'y dit rien.

Nous pouvons dire, pour conclure, qu'Atiq Rahimi met en lumière la souffrance de la femme "quelque part en Afghanistan ou ailleurs."125 L'éclairage porte sur la terrible condition de la femme afghane, comparable à n'importe quelle autre femme subissant le même sort dans le monde selon l'auteur lui-même. Celui-ci propose ici un thème difficile à aborder dans la culture afghane musulmane: il s'agit d'une solidarité utopique avec l'être méconnu, même plus méprisé, qu'est la femme.

Bref, Atiq Rahimi a écrit un roman à tendance réaliste qui a montré le malaise féminin à un moment où l'Afghanistan n'est pas arrivé à franchir le seuil de la modernité. Pour ce, "évidemment on ne peut que saluer une œuvre aussi audacieuse que féministe." 126

\section{Bibliographie sélective}

\section{$\underline{\text { I-Corpus }}$}

- $\quad$ Atiq Rahimi, Syngué sabour. Pierre de patience, Paris, P.O.L., 2008.

\section{$\underline{\text { II-Autres œuvres d'Atiq Rahimi }}$}

- $\quad$ Atiq Rahimi, Le Retour imaginaire, traduit par Sabrina Nouri, Paris, P.O.L., 2005.

- $\quad$ Atiq Rahimi, Les Mille maisons du rêve et de la terreur, traduit par Sabrina Nouri, Paris, P.O.L., 20002.

- $\quad$ Atiq Rahimi, Terre et cendres ["Khakestar-O-Khak"], traduit par Sabrina Nouri, P, Paris.O.L., 2000.

\section{III-Autres œuvres citées}

- $\quad$ Tahar Ben Jelloun, Harrouda, Paris, Denoël, 1973.

\section{$\underline{\text { IV-Ouvrages, articles et entretiens sur Atiq Rahimi }}$}

- $\quad$ Annick Cojean "Atiq Rahimi: roman d'un exilé", Le Monde, 6 decembre 2008, URL: https:/www.lemonde.fr/culture/article/2008/12/06/atiq-rahimi-roman-d-unexile_1127669_3246.html. (Consulté le 30/10/ 2017).

- "Atiq Rahimi", in: Wikipédia, the free encyclopedia, URL: https://en.wikipedia.org/wiki/Atiq_Rahimi. (Consulté le 30/10/ 2017).

Claire Mélanie, "Entretien exclusif avec l'écrivain et cinéaste Atiq Rahimi - seconde partie", jeudi 30 mai 2013, in: Eclairement, URL: http://www.eclairement.com/Entretienexclusif-avec-l-ecrivain,3406. (Consulté le 31/10/ 2017).

- $\quad$ Cinécution (publié par), "Cinécution: SYNGUE SABOUR - Atiq Rahimi - 2012", lundi 18 février 2013, URL: http://cinecution.blogspot.com/2013/02/syngue-sabour-atiqrahimi-2013.html. (Consulté le 30/10/ 2017). 
- "CR68 - Syngué sabour, pierre de patience - Atiq Rahimi", URL: http://doelan.blogspirit.com/archive/2009/01/15/cr68-syngue-sabour-pierre-de-patienceatiq-rahimi.html. (Consulté le 31/10/ 2017).

- $\quad$ Cyndie Boyer, "Atiq RAHIMI, Syngué sabour", in: littexpress, 5 janvier 2011, URL: http://littexpress.over-blog.net/article-atiq-rahimi-syngue-sabour-64120794.html (Consulté le 30/10/ 2017).

- $\quad$ Elodie Bernard (Entretien réalisé par), "Rencontre avec Atiq Rahimi", in: La Revue de Téhéran, $\mathrm{N}^{\circ}$ 39, février 2009, URL: http://www.teheran.ir/spip.php?article898. (Consulté le $31 / 10 / 2017$ ).

- Gabriella KÖRÖMI, "La symbolique des rideaux dans Syngué sabour d'Atiq Rahimi",URL:https://www.researchgate.net/.../305913071 La symbolique des rideaux d ans Syngue Sabour d'Atiq Rahimi, pp. 169-175.

- Jean-Louis Jeannelle, "Entretien avec Jean-Claude Carrière et Atiq Rahimi", In: Revue critique de fixxion française contemporaine, 2012, URL: http://www.revue-critique-defixxion-francaise contemporaine.org/rcffc/article/view/fx07.13/772. (Consulté le 31/10/ 2017).

- José Domingues de Almeida, «Écriture au féminin par procuration. Pierre de patience d'Atiq Rahimi », Intercâmbio, $\mathrm{n}^{\circ}$ 2, $2^{\mathrm{a}}$ série, 2009, pp. 7-18.

- Julia Piaton, "Atiq Rahimi, un conteur révolté", in: France-Amérique, The best of french culture, Février, 2011, URL: https://france-amerique.com/fr/atiq-rahimi-unconteur-revolte/. (Consulté le 30/10/ 2017).

- $\quad$ Martine Laval "Propos recueillis par", "Atiq Rahimi: "Je ne crains pas de dire la barbarie ou la décadence"", Grand Entretien, Publié le 21/11/2008, in: Télérama, URL: http://www.telerama.fr/livre/atiq-rahimi-je-ne-crains-pas-de-dire-la-barbarie-ou-la decadence,36049.php. (Consulté le 30/10/ 2017).

- $\quad$ Michel Nareau, "Atiq Rahimi : Un regard distancié", in: Nuit blanche, Numéro 126, Printemps 2012, pp. 38-42.

- $\quad$ Profizi, Alexandra. « Atiq Rahimi : "En Afghanistan, l'individu n'existe pas” ». La règle $d u$ jeu, Mis en ligne le 12 avril 2013, URL: http://aregledujeu.org/2013/04/12/13017/atiq-rahimi-en-afghanistanlindividu-nexiste-pas/.

(Consulté le 30/10/ 2017).

- $\quad$ Selenie, "Syngué Sabour - pierre de patience (2013) de Atiq Rahimi", 26 Février 2013, URL: http://www.selenie.fr/article-syngue-sabour-pierre-de-patience-2013-de-atiqrahimi-115698862.html. (Consulté le 31/10/ 2017).

\section{V-Ouvrages théoriques généreux}

Frantz Fanon, Sociologie d'une révolution, Paris, éd. Maspéro, 1975. 


\section{وضع المر أة المتردى فح رواية "سبينجيه صبور. حجر البحثبر " للكاتب الفرانكو-أفغانى عنيق رحيمى}

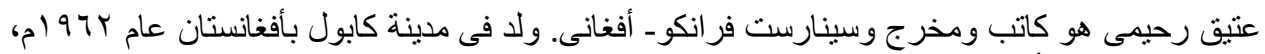

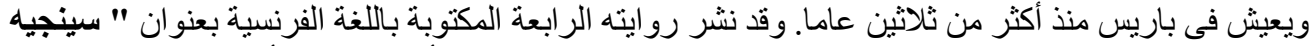

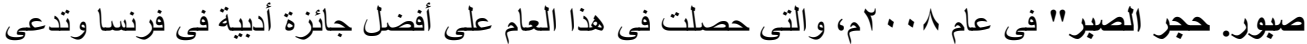



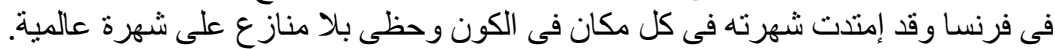



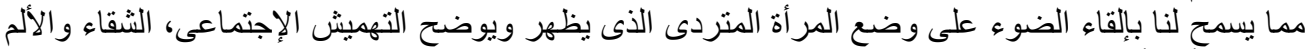



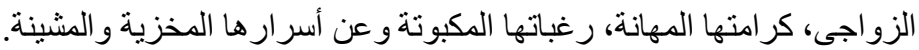

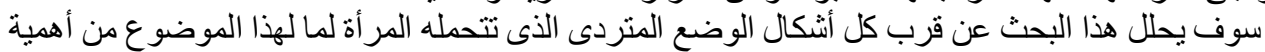

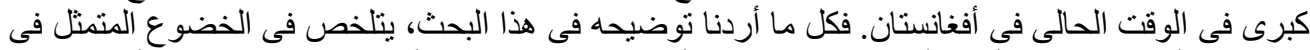

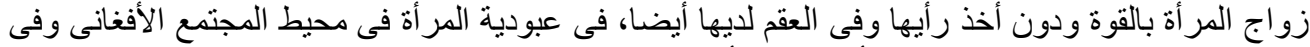

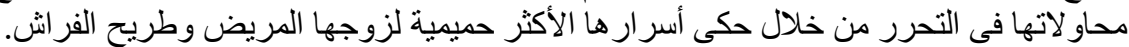

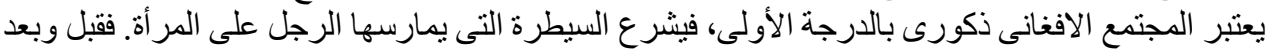

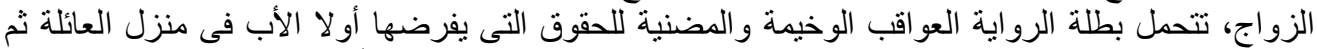

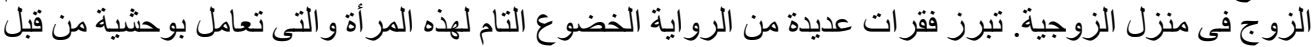

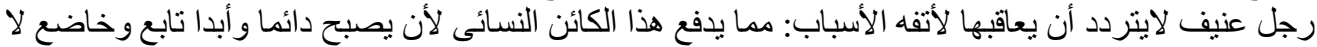

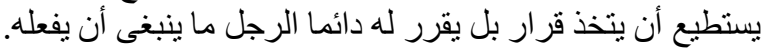

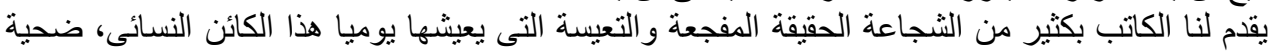



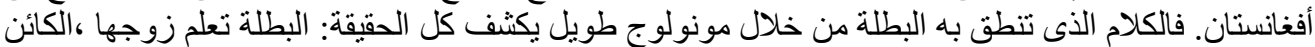

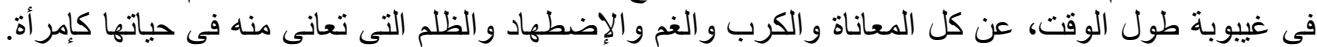

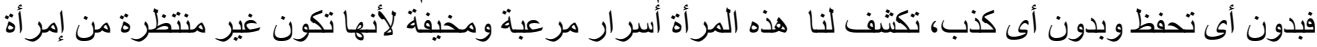
عاشت منر هبة ومحبوسة طيلة حياتها.

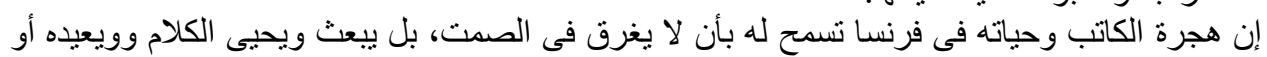



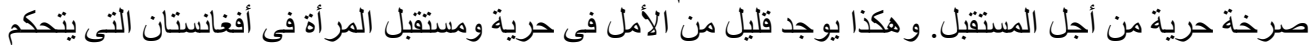

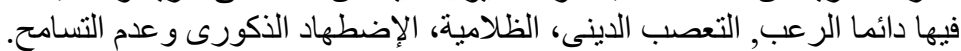

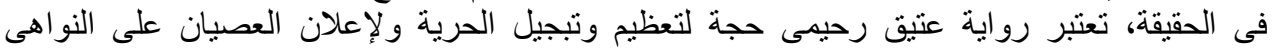



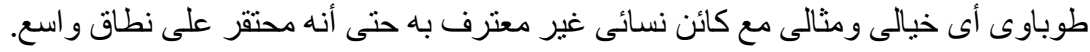

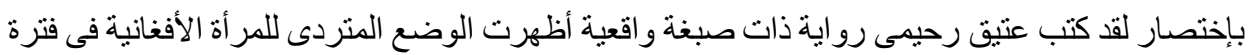

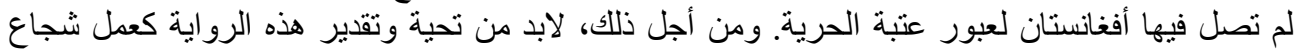
وجرىئ بقدر ما هو نسائى. 
${ }^{1} 1$ Annick Cojean "Atiq Rahimi: roman d'un exilé", Le Monde, 6 decembre 2008, URL: https://www.lemonde.fr/culture/article/2008/12/06/atiq-rahimi-roman-d-unexile 1127669 3246.html, (Consulté le 30/10/ 2017), p.3.

2 Elodie Bernard (Entretien réalisé par), "Rencontre avec Atiq Rahimi", in: La Revue de Téhéran, № 39, février 2009, URL: http://www.teheran.ir/spip.php?article898, p.2. (Consulté le 31/10/ 2017).

3 Atiq Rahimi, Terre et cendres ["Khakestar-O-Khak"], traduit par Sabrina Nouri, Paris, P.O.L., 2000.

4 Atiq Rahimi, Les Mille maisons du rêve et de la terreur, traduit par Sabrina Nouri, Paris, P.O.L., 20002.

5 Atiq Rahimi, Le Retour imaginaire, traduit par Sabrina Nouri, Paris, P.O.L., 2005.

${ }^{6}$ Atiq Rahimi, Syngué sabour. Pierre de patience, Paris, P.O.L., 2008. Dans le présent travail, les citations tirées de ce roman seront suivies du titre abrévié S.S. et du numéro de la page.

${ }^{7}$ Michel Nareau, "Atiq Rahimi : Un regard distancié", in: Nuit blanche, Numéro 126, Printemps 2012, pp. 38-42, p.38.

${ }^{8}$ Tiré de l'extrait "Atiq Rahimi", in: Wikipédia, the free encyclopedia, URL: https://en.wikipedia.org/wiki/Atiq Rahimi, p.3. (Consulté le 30/10/ 2017).

9 S.S., p.7.

${ }^{10}$ Ce point sera analysé en détails plus loin.

${ }^{11}$ Nous y reviendrons un peu plus tard.

12 S.S., p.13.

${ }^{13}$ José Domingues de Almeida, «Écriture au féminin par procuration. Pierre de patience d'Atiq Rahimi », Intercâmbio, $\mathrm{n}^{\mathrm{o}} 2$ 2, $2^{\mathrm{a}}$ série, 2009, pp. 7-18.

${ }^{14}$ Ibid, p.14.

${ }^{15}$ Elle n'était pas tuée et enterée vivante.

16 S.S., p.73.

17 Ibid, p72.

${ }^{18}$ Ibid, p.74.

${ }^{19}$ Ibid.

${ }^{20}$ Idem.

${ }^{21}$ Idem.

${ }^{22}$ Ibid, p.68.

${ }^{23}$ Ibid, p.69.

${ }^{24}$ Ibid, p.43.

${ }^{25}$ Ibid, p.69.

${ }^{26}$ Ibid, p.71.

${ }^{27}$ Ibid.

${ }^{28}$ Ibid.

${ }^{29}$ Idem.

${ }^{30}$ Idem.

31 Ibid, p.92. voir aussi pp.63, 77.

32 Ibid, p.15.

33 Frantz Fanon, Sociologie d'une révolution, Paris, éd. Maspéro, 1975, p.17.

34 S.S., p.72. 
35 Ibid, p.81.

${ }^{36}$ Le thème de la guerre sera traité un peu plus tard.

37 S.S, p.102.

38 Ibid.

39 Idem.

${ }^{40}$ Cyndie Boyer, "Atiq RAHIMI, Syngué sabour", in: littexpress, 5 janvier 2011, URL: http://littexpress.over-blog.net/article-atiq-rahimi-syngue-sabour-64120794.html, $\quad$ P.2 (Consulté le 30/10/ 2017).

41 S.S., pp: 102-103.

42 Ibid, p.104.

43 Ibid, p. 150.

44 Ibid, p.81.

45 Ibid, p.28.

46 Ibid.

47 Ibid, p.47.

48 Ibid, p.14.

49 Ibid, p.18.

50 Ibid, p.34.

${ }^{51}$ Cinécution (publié par), "Cinécution: SYNGUE SABOUR - Atiq Rahimi - 2012", lundi 18 février 2013, URL: http://cinecution.blogspot.com/2013/02/syngue-sabour-atiq-rahimi2013.html, P.1. (Consulté le 30/10/ 2017).

52 S.S., p.58.

53 Ibid, 31. Voir aussi pp: 17, 24-25, 86-87, 125-126.

54 Ibid, p.23.

55 Ibid, p.30.

56 Ibid, p.22.

57 Ibid, p.20. Voir aussi pp: 17-18, 21, 30.

58 Ibid, p. 21

59 Cyndie Boyer, op.cit., P. 2.

${ }^{60}$ Gabriella KÖRÖMI, "La symbolique des rideaux dans Syngué sabour d'Atiq Rahimi", URL: https://www.researchgate.net/.../305913071 La symbolique des rideaux dans Syngu e Sabour d'Atiq Rahimi, pp. 169-175, p.172.

61 S.S., p.92.

62 Ibid, p.104. Voir aussi: 46, 111, 125.

63 Ibid, p.46.

64 Ibid, p.63.

65 Ibid, p.47.

66 Ibid, p.28.

67 Ibid, pp:65-66.

68 Ibid, p.105.

69 Ibid, p.65.

70 Ibid, p.95.

${ }^{71}$ Profizi, Alexandra. « Atiq Rahimi : "En Afghanistan, l'individu n'existe pas” ». La règle du jeu, Mis en ligne le 12 avril 2013, URL: http://aregledujeu.org/2013/04/12/13017/atiqrahimi-en-afghanistanlindividu-nexiste-pas/, p.6. (Consulté le 30/10/ 2017).

72 S.S., p.28. 
73 Ibid, p.28.

74 Ibid, p. 91.

75 Gabriella KÖRÖMI, op.cit., P.173.

76 S.S., p. 27.

77 Ibid, p.34.

78 Ibid, p.32.

79 Ibid, p.24.

80 Ibid, p.64.

81 Ibid, p.67.

82 Ibid, p.66.

83 Ibid. p.30.

84 José Domingues de Almeida, op.cit., .13.

85 Cyndie Boyer, op.cit., p.2.

${ }^{86}$ S.S., p.76.

${ }^{87}$ Selenie, "Syngué Sabour - pierre de patience (2013) de Atiq Rahimi", 26 Février 2013, URL: http://www.selenie.fr/article-syngue-sabour-pierre-de-patience-2013-de-atiq-rahimi115698862.html, , p.1. (Consulté le 31/10/2017).

88 Julia Piaton, "Atiq Rahimi, un conteur révolté", in: France-Amérique, The best of french culture, Février, 2011, URL: https://france-amerique.com/fr/atiq-rahimi-un-conteurrevolte/, P.2 (Consulté le 30/10/2017).

89 Jean-Louis Jeannelle, "Entretien avec Jean-Claude Carrière et Atiq Rahimi", In: Revue critique de fixxion française contemporaine, 2012, URL: http://www.revue-critique-defixxion-francaise contemporaine.org/rcffc/article/view/fx07.13/772, p.4. (Consulté le 31/10/ 2017).

90 S.S., pp:26-27.

91 Ibid, p.65.

92 Ibid, p.27.

93 Ibid, p.66.

${ }^{94}$ Ibid, p.67.

${ }^{95}$ Tiré de l'extrait "CR68 - Syngué sabour, pierre de patience - Atiq Rahimi", URL: http://doelan.blogspirit.com/archive/2009/01/15/cr68-syngue-sabour-pierre-depatience-atiq-rahimi.html, P.1. (Consulté le 31/10/2017). S.S., p.42.

97 Ibid, p.87.

98 Ibid.

${ }^{99}$ Ibid, p.88.

100 José Domingues de Almeida, op. cit., P.13.

101 S.S., pp. 90-91.

102 Elodie Bernard, op.cit.,P.3.

103 S.S., pp.: 116-121

104 Ibid, pp. 95-98.

105 Ibid, pp.: 126, 135.

106 Ibid, p.129.

107 Ibid, p.130.

108 S.S., p. 150.

109 "Tout le monde croyait que c'etait moi qui etais sterile." Ibid.

110 Idem. 
111 Idem.

112 Tahar Ben Jelloun, Harrouda, roman, Paris, Denoël, 1973, p.81.

113 S.S., pp. 153-154.

114 Ibid, p.154.

115 Ibid.

116 Ibid, pp.154-155.

117 Elodie Bernard, op.cit., P.2.

118 José Domingues de Almeida, op.cit., P.11.

${ }^{119}$ Ibid., p.17.

120 S.S., p.13.

121 Ibid, p.155.

122 Martine Laval "Propos recueillis par", "Atiq Rahimi: "Je ne crains pas de dire la barbarie ou la décadence"", Grand Entretien, Publié le 21/11/2008, in: Télérama, URL: http://www.telerama.fr/livre/atiq-rahimi-je-ne-crains-pas-de-dire-la-barbarie-ou-la decadence,36049.php, p.6. (Consulté le 30/10/ 2017).

${ }_{123}$ Profizi, Alexandra, op.cit., P.8.

124 Claire Mélanie, "Entretien exclusif avec l'écrivain et cinéaste Atiq Rahimi - seconde partie", jeudi 30 mai 2013, in: Eclairement, URL: http://www.eclairement.com/Entretien-exclusif-avec-l-ecrivain,3406, p.2. (Consulté le 31/10/ 2017).

125 S.S., p.11.

126 Seleine, op.cit., p.1. 